\title{
Nuclear reprogramming
}

\section{Richard P. Halley-Stott ${ }^{1,2, *}$, Vincent Pasque ${ }^{3}$ and J. B. Gurdon ${ }^{1,2}$}

\section{Summary}

There is currently particular interest in the field of nuclear reprogramming, a process by which the identity of specialised cells may be changed, typically to an embryonic-like state. Reprogramming procedures provide insight into many mechanisms of fundamental cell biology and have several promising applications, most notably in healthcare through the development of human disease models and patient-specific tissue-replacement therapies. Here, we introduce the field of nuclear reprogramming and briefly discuss six of the procedures by which reprogramming may be experimentally performed:

\footnotetext{
${ }^{1}$ The Wellcome Trust/Cancer Research UK Gurdon Institute, University of Cambridge, Tennis Court Road, Cambridge, CB2 1QN, UK. ${ }^{2}$ Department of Zoology, University of Cambridge, Downing Street, Cambridge, CB2 3EJ, UK. ${ }^{3}$ University of California Los Angeles, Department of Biological Chemistry, Eli and Edythe Broad Center of Regenerative Medicine and Stem Cell Research, 615 Charles E. Young Drive South, BSRB 390D, Los Angeles, CA 90095, USA.
}

nuclear transfer to eggs or oocytes, cell fusion, extract treatment, direct reprogramming to pluripotency and transdifferentiation.

Key words: Transdifferentiation, Cell fusion, Induced pluripotency, Nuclear transfer, Pluripotent, Reprogramming

\section{Introduction}

The differentiated state of somatic cells is considered to be highly stable. During embryogenesis, developmentally plastic stem cells become specified, committed and ultimately restricted to particular cellular lineages in a highly coordinated manner. Once differentiated, somatic cells very rarely change from one differentiation state to another. When this does occur, however, it is often associated with disease and particularly cancer formation. The differentiated state of a somatic cell may be reversed experimentally to that of another cell type by a process termed 'nuclear reprogramming' (Gurdon and Melton, 2008). Nuclear reprogramming can most simply be defined as 'a process by which the differentiation state of a cell is changed to that of another state'. This may involve a differentiated cell reverting to a state of

\section{Development}

\section{Nuclear reprogramming}

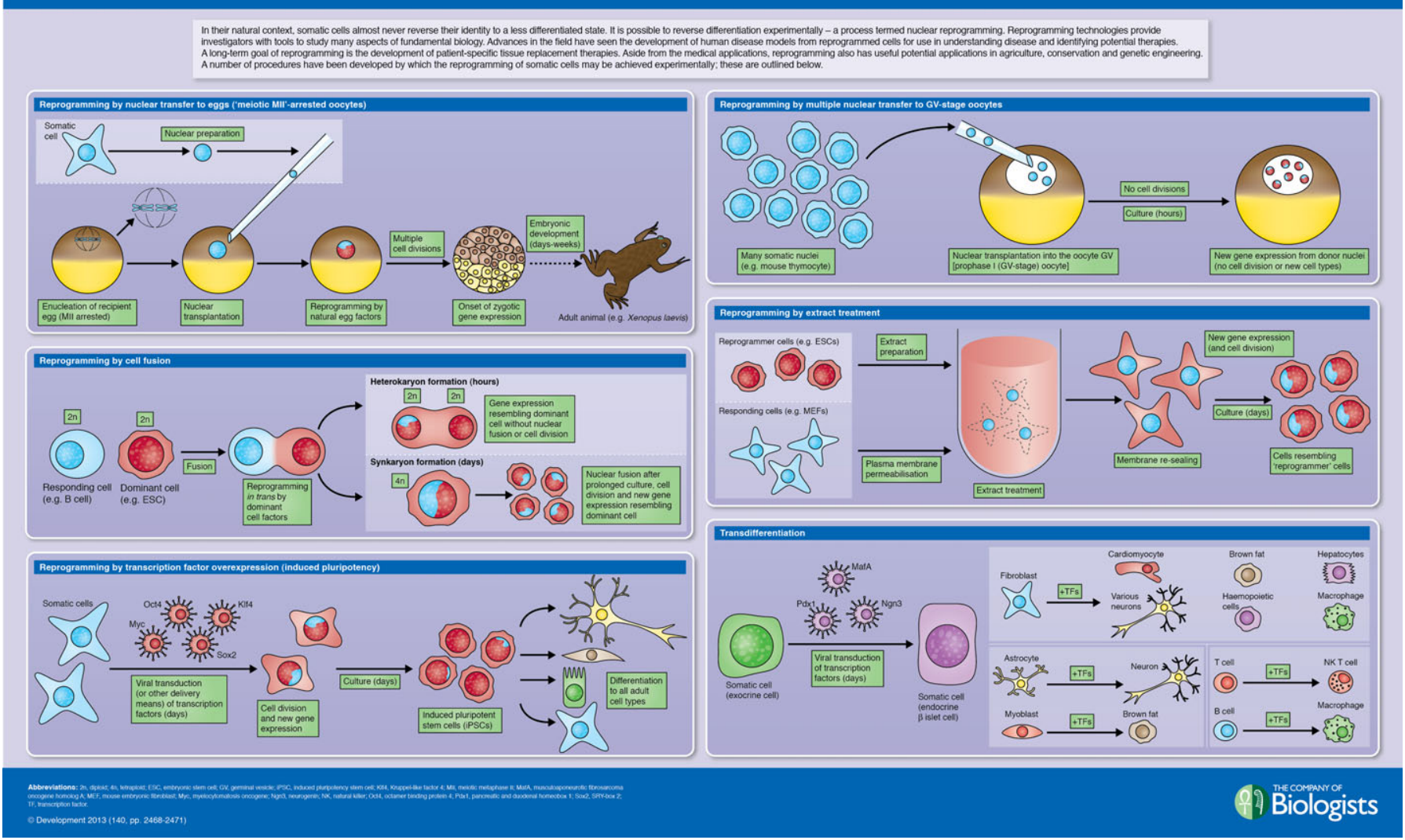


increased developmental plasticity ('de-differentiation'), or a switch from one differentiated cell type to another ('transdifferentiation' or 'direct reprogramming'). In its most extreme form, terminally differentiated cells may be reprogrammed to a state of totipotency, so that they are then capable of developing into a new adult animal.

The identity of any cell is determined by the expression of the lineage-specific genes that confer cellular identity (cellular phenotype). Consequently, in order to change a particular identity, a cell has to turn on new lineage-specific genes and turn off the old ones. As such, changes in transcription patterns are core to successful reprogramming, as is also the case with normal development. Reprogramming of somatic cells therefore involves resetting the epigenetic mechanisms that maintain stable gene expression, such as DNA methylation and histone modifications (Pasque et al., 2011). As well as these changes in gene expression profiles, successful nuclear reprogramming involves many other fundamental changes in cellular function, such as DNA replication, nuclear organisation, cell division and metabolism. The experimental induction of reprogramming gives researchers a window to understand these fundamental aspects of cell biology.

In addition to using reprogramming technologies as a tool to understand fundamental biology, the reprogramming of somatic cells carries significant potential medical value. Recent advances in the reprogramming field have led to the development of a number of human disease models, generated from the reprogramming of diseased somatic cells (Wu and Hochedlinger, 2011; Yamanaka, 2012). These will undoubtedly be invaluable in understanding and combating a number of human diseases. The use of reprogrammed human cells also provides an ethically attractive source of autologous human tissue for cell replacement therapies ( $\mathrm{Wu}$ and Hochedlinger, 2011).

This article will highlight the range of techniques by which nuclear reprogramming may be studied by experimental induction in the laboratory.

\section{Nuclear reprogramming by nuclear transfer to eggs (meiotic metaphase II oocytes)}

Nuclear transfer involves the physical transplantation of a single nucleus into a meiotic metaphase II arrested egg (or 'MII oocyte' in the mammalian field), usually after removing the recipient egg's genetic material. It was by this method that the first cloning of amphibians and later of mammals was performed (Gurdon et al., 1958; Wakayama et al., 1998; Wilmut et al., 1997) and is often called 'somatic cell nuclear transfer' (SCNT). This procedure was initially used to establish the concept of genome conservation during development (Briggs and King, 1952; Gurdon et al., 1958). These early experiments also demonstrated that the cloning of an animal was possible. Nuclear transfer has been successfully used to reprogramme many different species (Byrne et al., 2007; Cibelli, 2007; Noggle et al., 2011; Wakayama and Yanagimachi, 1999) and much of our understanding of reprogramming comes from experiments using this system (reviewed by Gurdon and Wilmut, 2011). The cloned embryonic cells resulting from the procedure may also be harvested and cultured in vitro. By this means, it is possible to derive embryonic stem cells (ESCs) from cloned mammalian embryos (Byrne et al., 2007; Noggle et al., 2011; Yang et al., 2007). Such cultured embryonic cells may also be differentiated into various adult cell types (Wakayama et al., 2001).

Nuclear reprogramming by this route is thought to 'mimic' natural fertilisation; the oocyte attempts to reprogramme the incoming somatic nucleus in much the same way as it reprogrammes the paternal genome after fertilisation (Gao et al.,
2007). Reprogramming by SCNT is mediated by natural components of the egg and involves extensive DNA replication and cell division (Jullien et al., 2011). This constantly changing nuclear landscape and the practical limitations of SCNT makes studying the early transcriptional changes involved in reprogramming somewhat difficult (Jullien et al., 2011).

The efficiency of SCNT, as judged by the generation of entirely normal adult animals, is low, below $\sim 1-2 \%$ when adult somatic nuclei are used as donors (Yang et al., 2007). Additionally, some clones may display a number of abnormalities, phenotypically and at a molecular and physiological level (Wilmut et al., 2002; Yang et al., 2007). The low efficiency and abnormalities are likely to be attributable to a failure to completely reprogramme the donor genome, reflecting the 'stability of differentiation' seen in adult cells. This is illustrated by the phenomenon of 'epigenetic memory', in which a 'memory' of the donor cell gene expression is retained by some cells of the resulting cloned embryos. This has been shown to be in part due to incomplete resetting of epigenetic factors, such as DNA methylation and histone variants, in the donor nucleus $(\mathrm{Ng}$ and Gurdon, 2007).

\section{Direct transcriptional reprogramming by nuclear transfer to prophase I amphibian oocytes}

A modified form of SCNT involves transplanting multiple somatic nuclei into the enlarged nucleus [termed the germinal vesicle $(\mathrm{GV})$ ] of developing amphibian oocytes. These large immature oocytes are arrested in meiotic prophase I and are undergoing intense production of the maternal transcripts and proteins that will be necessary for supporting early embryonic development until zygotic genome activation (Gao et al., 2002).

There is no DNA replication or cell division by this reprogramming route and, as such, no new cell types are generated. There is, however, considerable transcriptional reactivation of certain silent genes within a few hours (Halley-Stott et al., 2010). By this procedure, silent genes [such as the pluripotency genes Oct4 (Pou5f1) and Sox2] in the somatic donor nuclei become directly activated, in the absence of cell division and protein synthesis (Halley-Stott et al., 2010). The oocyte will transcriptionally reprogramme nuclei from another species (mouse, for example), allowing newly synthesised transcript to be distinguished from the maternal content of the oocyte by sequence identity.

Using this system, it is possible to explore the conditions that are required to promote the activation of silent genes, a process that may be referred to as 'transcriptional reprogramming'. The large size of the Xenopus oocyte and the ease of manipulating endogenous reprogramming factors by overexpression or knockdown make this system a powerful tool for studying reprogramming in a purely transcriptional context.

\section{Reprogramming by cell fusion}

Transcriptional changes in somatic cells can be achieved by fusing the plasma membranes of different cell types. The use of embryonic 'dominant' cells, such as ESCs, to reprogramme somatic cells can induce the transcription of pluripotency-associated genes under the correct conditions (Yamanaka and Blau, 2010). Upon fusion, the nuclei of both cell types can remain separate in the common cytoplasm (heterokaryon formation) or, after mitosis, fuse themselves to form a hybrid genome (synkaryon). In the case of early heterokaryons, there is thought to be no cell division and a direct transactivation of genes in the responding cell (Chiu and Blau, 1984), although the role of DNA synthesis during heterokaryonbased reprogramming is controversial (Tsubouchi et al., 2013). By 
contrast, synkaryons undergo DNA replication and cell division, forming a hybrid genome with a mixture of genetic material from both starting nuclei, although often chromosomes from one of the genomes are lost (Yamanaka and Blau, 2010).

As in the case of nuclear reprogramming with GV-stage oocytes, heterokaryon reprogramming may also be performed within or across species (Yamanaka and Blau, 2010). The latter case permits researchers to easily follow transcriptional changes in the responding nucleus without contributing signals from 'carry-over' transcript derived from the dominant cell. Moreover, the ability to manipulate both the responding and dominant cells using standard cell culture techniques makes cell fusion a useful technique for studying the mechanisms that underpin the early stages of reprogramming (Piccolo et al., 2011).

\section{Reprogramming by extract treatment}

Reprogramming may also be achieved, at least in part, by exposing permeabilised cells to protein extracts that are prepared, for example, from pluripotent cells (Taranger et al., 2005). The plasma membranes of these permeabilised cells may then be re-sealed and the cells cultured, leading to the expression of previously silent genes. This reprogramming is thought to be associated with cell division and DNA replication in most instances. As with the other reprogramming procedures, the efficiency of extract-based reprogramming appears to be low (Bru et al., 2008).

Extract treatment as a reprogramming system is relatively less developed than other systems. However, an appealing aspect of reprogramming by this means is the ability to biochemically introduce or deplete reprogramming factors to or from the extract. Furthermore, the use of extract provides an opportunity for biochemical fractionation, potentially allowing investigators to identify novel reprogramming factors (Singhal et al., 2010). Extracttreated cells may also be subsequently used as starting material for induced pluripotency or SCNT experiments (Bui et al., 2012; Ganier et al., 2011). Extracts prepared from relatively differentiated cells may also be used to 'transdifferentiate' cells, at least in part (Håkelien et al., 2002). Reproducibility of extract treatments is a concern in the field (Liu et al., 2011; Singhal et al., 2010). This is probably due to the difficulty of preparing high quality extracts, which might be solved as this system develops.

\section{Reprogramming by transcription factor overexpression (induced pluripotency)}

The most recent major advance in reprogramming was the discovery that cells could be reprogrammed by the overexpression of key transcription factors (Takahashi and Yamanaka, 2006). Four transcription factors (Oct4, Sox2, Klf4 and Myc) can be virally transfected into somatic cells and, under the correct cell culture conditions, will reprogramme some of them to an ESC-like state, generating what are termed 'induced pluripotent stem cells' (iPSCs) (Yamanaka, 2012).

Reprogramming by this route drives the induction of pluripotent gene expression profiles reminiscent of ESCs, while largely silencing somatic gene expression. There is extensive DNA replication and cell division leading to the generation of cells of this new identity (Koche et al., 2011). These cells may then be differentiated into any cell type of the adult body (Takahashi and Yamanaka, 2006).

Research on iPSCs has exploded in the last 5 years with a number of technological advancements and mechanistic insights coming to the fore (Papp and Plath, 2013). A significant portion of the work has focused on enhancing the low efficiency of induced pluripotency-based reprogramming, which is thought result at least partially from the repressive nature of chromatin at repressed loci in somatic cells and the stochastic nature of the procedure (Hanna et al., 2009; Jullien et al., 2011; Papp and Plath, 2013).

In addition, a large amount of research has been directed at generating iPSCs without the use of the oncogene $M y c$ and viral gene delivery vehicles, in order to eliminate potential oncogenesis (Nakagawa et al., 2008; Yamanaka, 2012). The primary drive behind this work is to develop systems to generate iPSCs in sufficient numbers so that they may be safely used for tissue replacement therapies (Okita and Yamanaka, 2011).

As with other reprogramming systems, there also appears to be a degree of epigenetic memory in iPSCs, with reprogrammed cells displaying epigenetic signatures related to the original cell type from which they were reprogrammed (Graf, 2009). Efforts are being made to overcome this and there is no reason why this should limit the medical application of this technology in the future.

\section{Transdifferentiation by transcription factor overexpression (direct programming)}

An exciting recent branch of reprogramming encompasses 'transdifferentiation' or 'transversion', whereby a somatic cell is switched from one identity to another, but not necessarily to one of increased developmental plasticity. The use of a cocktail of transcription factors has been successfully used to transdifferentiate a number of cell types from one lineage to that of another (Graf, 2011; Vierbuchen and Wernig, 2012).

Such reprogramming may occur within a lineage, such as the conversion of an exocrine cell to an endocrine cell (both endoderm derived) (Zhou et al., 2008) or alternatively across lineages, as has been demonstrated by the conversion of fibroblasts to neurons (mesoderm-to-ectoderm lineage switch) (Vierbuchen et al., 2010). It can be expected that the list of cell types that can be subject to transdifferentiation will continue grow as new combinations of transcription factors, able to confer distinct cellular identities, are identified (Vierbuchen and Wernig, 2011).

Transdifferentiation also promises a number of potential advantages for the generation of clinically relevant cells, particularly with respect to the efficiency of conversion, which appears to be high in some cases. Additionally, cells derived by this route may carry less risk of generating carcinomas because oncogenes need not be used in the reprogramming procedure (Sancho-Martinez et al., 2012).

\section{Perspectives}

Over the past six decades, nuclear reprogramming has acquired ever-increasing momentum, fuelled by the realisation that it could help to alleviate human suffering as well as uncover fundamental biological principles.

Despite the many major advances in the reprogramming field made in recent years, there are still a number of challenges facing investigators. Key outstanding questions include: What are the molecular mechanisms of nuclear reprogramming? Why is the efficiency of the process so low? What factors and mechanisms maintain the stable state of cellular identity? In each system, what are the steps of nuclear reprogramming? Does reprogramming by induced pluripotency or transdifferentiation involve a gradual reversion of the developmental steps followed during embryogenesis? What can we learn about the mechanisms of diseases using reprogrammed cells from patients? And most importantly, can we design effective new cures starting from a patient's own cells and reprogramme these to therapeutically useful 
cells? Progress so far and the development of modern laboratory techniques provide a battery of invaluable tools to address these outstanding questions and the field can expect many more exciting fundamental and applied discoveries in the coming decades.

\section{Acknowledgements}

The authors would like to thank Dorit Hockman for comments and critical reading of the manuscript.

\section{Funding}

R.P.H.-S. and J.B.G. are supported by the Medical Research Council and the Wellcome Trust. V.P. acknowledges the support of a California Institute for Regenerative Medicine training grant for postdoctoral fellowship.

\section{Competing interests statement}

The authors declare no competing financial interests.

\section{Development at a Glance}

A high-resolution version of the poster is available for downloading in the online version of this article at http://dev.biologists.org/content/140/12/2468.full

\section{References}

Briggs, R. and King, T. J. (1952). Transplantation of living nuclei from blastula cells into enucleated frogs' eggs. Proc. Natl. Acad. Sci. USA 38, 455-463.

Bru, T., Clarke, C., McGrew, M. J., Sang, H. M., Wilmut, I. and Blow, J. J. (2008), Rapid induction of pluripotency genes after exposure of human somatic cells to mouse ES cell extracts. Exp. Cell Res. 314, 2634-2642.

Bui, H.-T., Kwon, D.-N., Kang, M.-H., Oh, M.-H., Park, M.-R., Park, W.-J., Paik,

S.-S., Van Thuan, N. and Kim, J.-H. (2012). Epigenetic reprogramming in somatic cells induced by extract from germinal vesicle stage pig oocytes. Development 139, 4330-4340.

Byrne, J. A., Pedersen, D. A., Clepper, L. L., Nelson, M., Sanger, W. G., Gokhale, S., Wolf, D. P. and Mitalipov, S. M. (2007). Producing primate embryonic stem cells by somatic cell nuclear transfer. Nature 450, 497-502.

Chiu, C. P. and Blau, H. M. (1984). Reprogramming cell differentiation in the absence of DNA synthesis. Cell 37, 879-887.

Cibelli, J. (2007). Developmental biology. A decade of cloning mystique. Science 316, 990-992.

Ganier, O., Bocquet, S., Peiffer, I., Brochard, V., Arnaud, P., Puy, A., Jouneau,

A., Feil, R., Renard, J.-P. and Méchali, M. (2011). Synergic reprogramming of mammalian cells by combined exposure to mitotic Xenopus egg extracts and transcription factors. Proc. Natl. Acad. Sci. USA 108, 17331-17336.

Gao, S., Gasparrini, B., McGarry, M., Ferrier, T., Fletcher, J., Harkness, L., De Sousa, P. and Wilmut, I. (2002). Germinal vesicle material is essential for nucleus remodeling after nuclear transfer. Biol. Reprod. 67, 928-934.

Gao, T., Zheng, J., Xing, F., Fang, H., Sun, F., Yan, A., Gong, X., Ding, H., Tang, F. and Sheng, H. Z. (2007). Nuclear reprogramming: the strategy used in normal development is also used in somatic cell nuclear transfer and parthenogenesis. Cell Res. 17, 135-150.

Graf, T. (2009). An uphill battle toward pluripotency. Nat. Genet. 41, 960-961.

Graf, T. (2011). Historical origins of transdifferentiation and reprogramming. Cell Stem Cell 9, 504-516.

Gurdon, J. B. and Melton, D. A. (2008). Nuclear reprogramming in cells. Science 322, 1811-1815.

Gurdon, J. B. and Wilmut, I. (2011). Nuclear transfer to eggs and oocytes. Cold Spring Harb. Perspect. Biol. 3, a002659.

Gurdon, J. B., Elsdale, T. R. and Fischberg, M. (1958). Sexually mature individuals of Xenopus laevis from the transplantation of single somatic nuclei. Nature 182, 64-65.

Håkelien, A.-M., Landsverk, H. B., Robl, J. M., Skålhegg, B. S. and Collas, P. (2002). Reprogramming fibroblasts to express T-cell functions using cell extracts. Nat. Biotechnol. 20, 460-466.

Halley-Stott, R. P., Pasque, V., Astrand, C., Miyamoto, K., Simeoni, I., Jullien, J. and Gurdon, J. B. (2010). Mammalian nuclear transplantation to germinal vesicle stage Xenopus oocytes - a method for quantitative transcriptional reprogramming. Methods 51, 56-65.

Hanna, J., Saha, K., Pando, B., van Zon, J., Lengner, C. J., Creyghton, M. P. van Oudenaarden, A. and Jaenisch, R. (2009). Direct cell reprogramming is a stochastic process amenable to acceleration. Nature 462, 595-601.

Jullien, J., Pasque, V., Halley-Stott, R. P., Miyamoto, K. and Gurdon, J. B. (2011). Mechanisms of nuclear reprogramming by eggs and oocytes: a deterministic process? Nat. Rev. Mol. Cell Biol. 12, 453-459.

Koche, R. P., Smith, Z. D., Adli, M., Gu, H., Ku, M., Gnirke, A., Bernstein, B. E. and Meissner, A. (2011). Reprogramming factor expression initiates widespread targeted chromatin remodeling. Cell Stem Cell 8, 96-105.
Liu, Y., Østrup, O., Li, J., Vajta, G., Kragh, P. M., Purup, S. and Callesen, H. (2011). Cell colony formation induced by Xenopus egg extract as a marker for improvement of cloned blastocyst formation in the pig. Cell. Reprogram. 13 521-526.

Nakagawa, M., Koyanagi, M., Tanabe, K., Takahashi, K., Ichisaka, T., Aoi, T., Okita, K., Mochiduki, Y., Takizawa, N. and Yamanaka, S. (2008). Generation of induced pluripotent stem cells without Myc from mouse and human fibroblasts. Nat. Biotechnol. 26, 101-106.

Ng, R. K. and Gurdon, J. B. (2007). Epigenetic memory of an active gene state depends on histone H3.3 incorporation into chromatin in the absence of transcription. Nat. Cell Biol. 10, 102-109.

Noggle, S., Fung, H.-L., Gore, A., Martinez, H., Satriani, K. C., Prosser, R., Oum, K., Paull, D., Druckenmiller, S., Freeby, M. et al. (2011). Human oocytes reprogram somatic cells to a pluripotent state. Nature $\mathbf{4 7 8}, 70-75$

Okita, K. and Yamanaka, S. (2011). Induced pluripotent stem cells: opportunities and challenges. Philos. Trans. R. Soc. Lond. B Biol. Sci. 366, 21982207.

Papp, B. and Plath, K. (2013). Epigenetics of reprogramming to induced pluripotency. Cell 152, 1324-1343.

Pasque, V., Jullien, J., Miyamoto, K., Halley-Stott, R. P. and Gurdon, J. B. (2011). Epigenetic factors influencing resistance to nuclear reprogramming Trends Genet. 27, 516-525.

Piccolo, F. M., Pereira, C. F., Cantone, I., Brown, K., Tsubouchi, T., Soza-Ried, J., Merkenschlager, M. and Fisher, A. G. (2011). Using heterokaryons to understand pluripotency and reprogramming. Philos. Trans. R. Soc. Lond. B Biol. Sci. 366, 2260-2265.

Sancho-Martinez, I., Baek, S. H. and Izpisua Belmonte, J. C. (2012). Lineage conversion methodologies meet the reprogramming toolbox. Nat. Cell Biol. 14 892-899.

Singhal, N., Graumann, J., Wu, G., Araúzo-Bravo, M. J., Han, D. W., Greber, B., Gentile, L., Mann, M. and Schöler, H. R. (2010). Chromatin-remodeling components of the baf complex facilitate reprogramming. Cell 141, 943-955.

Takahashi, K. and Yamanaka, S. (2006). Induction of pluripotent stem cells from mouse embryonic and adult fibroblast cultures by defined factors. Cell 126, 663-676

Taranger, C. K., Noer, A., Sørensen, A. L., Håkelien, A.-M., Boquest, A. C. and Collas, P. (2005). Induction of dedifferentiation, genomewide transcriptional programming, and epigenetic reprogramming by extracts of carcinoma and embryonic stem cells. Mol. Biol. Cell 16, 5719-5735.

Tsubouchi, T., Soza-Ried, J., Brown, K., Piccolo, F. M., Cantone, I., Landeira, D., Bagci, H., Hochegger, H., Merkenschlager, M. and Fisher, A. G. (2013), DNA synthesis is required for reprogramming mediated by stem cell fusion. Cell 152, 873-883.

Vierbuchen, T. and Wernig, M. (2011). Direct lineage conversions: unnatural but useful? Nat. Biotechnol. 29, 892-907.

Vierbuchen, T. and Wernig, M. (2012). Molecular roadblocks for cellular reprogramming. Mol. Cell 47, 827-838.

Vierbuchen, T., Ostermeier, A., Pang, Z. P., Kokubu, Y., Südhof, T. C. and Wernig, M. (2010). Direct conversion of fibroblasts to functional neurons by defined factors. Nature $\mathbf{4 6 3}, 1035-1041$

Wakayama, T. and Yanagimachi, R. (1999). Cloning of male mice from adult tail-tip cells. Nat. Genet. 22, 127-128.

Wakayama, T., Perry, A. C., Zuccotti, M., Johnson, K. R. and Yanagimachi, R. (1998). Full-term development of mice from enucleated oocytes injected with cumulus cell nuclei. Nature 394, 369-374.

Wakayama, T., Tabar, V., Rodriguez, I., Perry, A. C., Studer, L. and Mombaerts, P. (2001). Differentiation of embryonic stem cell lines generated from adult somatic cells by nuclear transfer. Science $\mathbf{2 9 2}, 740-743$.

Wilmut, I., Schnieke, A. E., McWhir, J., Kind, A. J. and Campbell, K. H. (1997) Viable offspring derived from fetal and adult mammalian cells. Nature $\mathbf{3 8 5}$ 810-813

Wilmut, I., Beaujean, N., de Sousa, P. A., Dinnyes, A., King, T. J., Paterson, L. A., Wells, D. N. and Young, L. E. (2002). Somatic cell nuclear transfer. Nature 419, 583-586

Wu, S. M. and Hochedlinger, K. (2011). Harnessing the potential of induced pluripotent stem cells for regenerative medicine. Nat. Cell Biol. 13, 497-505.

Yamanaka, S. (2012). Induced pluripotent stem cells: past, present, and future. Cell Stem Cell 10, 678-684.

Yamanaka, S. and Blau, H. M. (2010). Nuclear reprogramming to a pluripotent state by three approaches. Nature 465, 704-712.

Yang, X., Smith, S. L., Tian, X. C., Lewin, H. A., Renard, J.-P. and Wakayama, T. (2007). Nuclear reprogramming of cloned embryos and its implications for therapeutic cloning. Nat. Genet. 39, 295-302

Zhou, Q., Brown, J., Kanarek, A., Rajagopal, J. and Melton, D. A. (2008). In vivo reprogramming of adult pancreatic exocrine cells to $\beta$-cells. Nature $\mathbf{4 5 5}$ $627-632$. 